\title{
A pilot randomised control trial of the efficacy of stability-based training with visualisation for people with chronic ankle instability
}

\author{
L. Forsyth ${ }^{1} \cdot$ J. Bonacci ${ }^{2} \cdot$ C. Childs ${ }^{1}$
}

Received: 21 July 2021 / Accepted: 14 February 2022 / Published online: 5 March 2022

(c) The Author(s) 2022

\begin{abstract}
Chronic ankle instability (CAI) is associated with recurring symptoms that inhibit daily activity. Stability-based rehabilitative training is recommended for CAI. Visualisation (VIS) produces real-time feedback using motion capture and virtual reality. This pilot study aimed to determine the feasibility, adherence, safety, and efficacy of incorporating VIS into stability training for people with CAI. Efficacy was examined through effect of VIS training on dynamic stability, perception of stability, and rehabilitative experience. Individuals with CAI completed a 4-week stability-based training programme with VIS, or without visualisation (NO-VIS). Participants completed the Star Excursion Balance Test (SEBT) and Cumberland Ankle Instability Tool (CAIT) prior to, and after training. Enjoyment of training was recorded using the Physical Activity Enjoyment Scale (PACES-8). Of 17 participants (VIS = 10, NO-VIS =7), there were 2 drop outs (VIS = 1, NO-VIS =1). No adverse events were reported, and participant drop-out was due to injury unrelated to the study. The VIS group showed a significantly greater increase in average SEBT reach distance $(d=1.7, p=0.02)$. No significant differences were reported for the CAIT or PACES-8. This study supports the feasibility and safety of stability-based training with VIS in those with CAI. The enhanced performance outcome on the SEBT suggests VIS may enhance stability-based training.
\end{abstract}

Keywords Stability $\cdot$ Virtual reality $\cdot$ Feedback $\cdot$ Chronic ankle instability $\cdot$ Rehabilitation

\section{Introduction}

Chronic ankle instability (CAI) is a complicated multi-faceted clinical condition affecting $20-70 \%$ of those who have experienced an ankle sprain $[1,2]$. CAI is associated with recurrent ankle sprains, mechanical laxity, and/or perceived instability that inhibits daily activity and impacts quality of life [2]. CAI alters joint contact stress and distribution of loading of the articular surface [1, 3, 4]. A link between CAI and ankle post-traumatic osteoarthritis (PTOA) has been established [5]. The degree of physical impairment associated with ankle osteoarthritis is equivalent to that of end-stage kidney disease and congestive heart failure [6]. Surgical treatment includes ankle arthroplasty or arthrodesis [3]. However, fusion of the joint reduces motion, altering

L. Forsyth

lauren.forsyth@strath.ac.uk

1 Faculty of Biomedical Engineering, University of Strathclyde, Glasgow, UK

2 Centre for Sports Research, School of Exercise and Nutrition Science, Deakin University, Geelong, Australia stress on other joints. Successful outcomes for arthroplasty are low with a $42 \%$ revision rate and lower patient satisfaction [7, 8]. Given the current ankle PTOA treatment options, and that it is a younger population that are typically affected, emphasis should be placed on prevention and conservative management of CAI $[3,6]$.

Stability-based rehabilitative training is the most recommended rehabilitation strategy for people with CAI [4, 9]. Stability training should challenge the sensorimotor system under different task and environment conditions for optimal rehabilitation outcomes [10]. Examples include reducing the base of support, performing dynamic movements, disturbing centre of gravity, and cognitive and sensory manipulation [11-13].

Virtual reality (VR) presents an enhanced opportunity for interactive simulation using computer software to provide feedback of movement and performance. For stability-based training, this facilitates practice with externally focussed augmented feedback incorporating motor and cognitive manipulation in a safe environment $[10,14,15]$. VR interventions can also easily be adapted and individualised to the needs of the user [16]. A systematic review reported 
VR balance training to be equally as effective as traditional balance training for prevention and rehabilitation of musculoskeletal lower limb impairments [16]. No study has compared VR balance training to traditional balance training specifically for people with CAI.

VR training enhances stimulation and engagement and has been associated with greater satisfaction and enjoyment of training [17-19]. For those with musculoskeletal impairments, this has increased adherence to rehabilitation [20]. This may create more conducive conditions for rehabilitation since poor programme compliance can hinder a programme's effectiveness $[4,21]$. Compliance with exercise programs has been reported to be as low as $50 \%$, with reduced compliance over time [21, 22]. Low adherence to rehabilitation can be due to low interest or enjoyment in the exercises, negative emotional states, or perceived lack of value [23-25]. VR could be used to create an environment to motivate, monitor, and encourage compliance, thereby leading to more effective rehabilitation.

Visualisation is an emerging technique that connects biomechanical analysis and VR. Visualisation produces real-time feedback by accurately monitoring movement and progress in a diverse, challenging, and controllable environment, representative of real-world situations. Communication could therefore be improved between patient and specialist by making tasks easier to understand, promoting ownership of rehabilitation and intrinsic motivation, whilst enabling objective monitoring of progress [26].

The aim of this pilot study was to determine the feasibility, adherence, safety, and efficacy of incorporating visualisation into stability training for people with CAI. Efficacy was examined through the effect of visualisation on dynamic stability, perception of stability, and rehabilitative experience.

\section{Methods}

\subsection{Experimental design}

A pilot randomised-controlled trial was conducted to assess the feasibility of a stability-based training programme using visualisation for people with CAI. The study was approved by the University of Strathclyde and Deakin University ethics committee (DEC 2018.243) and received NHS R\&D approval for testing on an NHS site (IRAS project ID 247615).

\subsection{Participants}

Volunteers from local universities and surrounding communities were recruited via poster and social media advertisements and screened for CAI using the International Ankle
Consortium guidelines [27]. Participants were required to have experienced all of the following: (1) history of at least one significant ankle sprain, (2) most recent injury to have occurred more than 3 months prior to study participation, (3) history of previous episodes of 'giving way', recurrent sprain, or 'feelings of instability', and (4) perceived ankle instability determined by a score of $<24$ on the Cumberland Ankle Instability Tool (CAIT). The CAIT is a recognised tool for identifying CAI (intraclass correlation coefficient $\left.[\mathrm{ICC}]_{2,1}=0.96\right)[28]$. The CAIT contains nine items with a maximum score of 30. A lower score indicates a decreased perception of stability. A history of surgeries or fractures of either lower extremity meant participants were not eligible for participation. All participants provided written informed consent.

\subsection{Instrumentation and protocol}

All testing and training sessions were supervised and completed in one of three laboratories. The three sites used were (i) Human Performance Laboratory at Glasgow Royal Infirmary, Scotland, UK; (ii) SportScotland Institute of Sport, Scotland, UK; and (iii) Biomechanics laboratory at Deakin University, Geelong, Australia. Testing sessions were conducted the week prior to, and the week following completion of the training block. The training programme was completed biweekly over a 4-week period with visualisation (VIS) or training without visualisation (NO-VIS).

At the pre-training testing session, participant's age, mass, leg dominance, physical activity levels, sport participation, and ankle injury history were collected.

Each of the testing sites had motion capture systems for measuring biomechanical data (Vicon MX cameras sampling at $100 \mathrm{~Hz}$ through Vicon Tracker v3.5.1, Oxford, UK). Data was live-streamed into D-Flow software for the visualisation (Motek Medical, Amsterdam, the Netherlands). Body segments were determined using pointer calibration at 16 pre-determined anatomical landmarks and segment clusters (Strathclyde Cluster Model) which allowed for an avatar to be generated that showed the participants' pose in real time (Fig. 1) [29, 30]. Joint coordinate systems and kinematics for this model were calculated as per International Society of Biomechanics recommendations $[31,32]$.

\subsection{Stability-based training intervention}

The stability-based training programme was derived from standard practice and consistent with the principles of training [33-37]. The programme aimed to improve and challenge ankle joint stability and postural control by incorporating elements relative to activities of daily living, such as single leg standing, change of support, transfer of weight, 
Fig. 1 Cluster positions on each segment (left) and anatomical landmarks for calibration (middle) and the avatar after calibration (right)

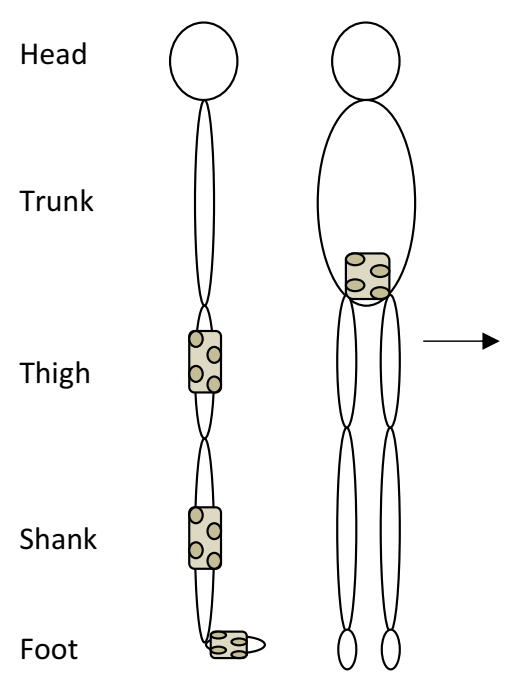

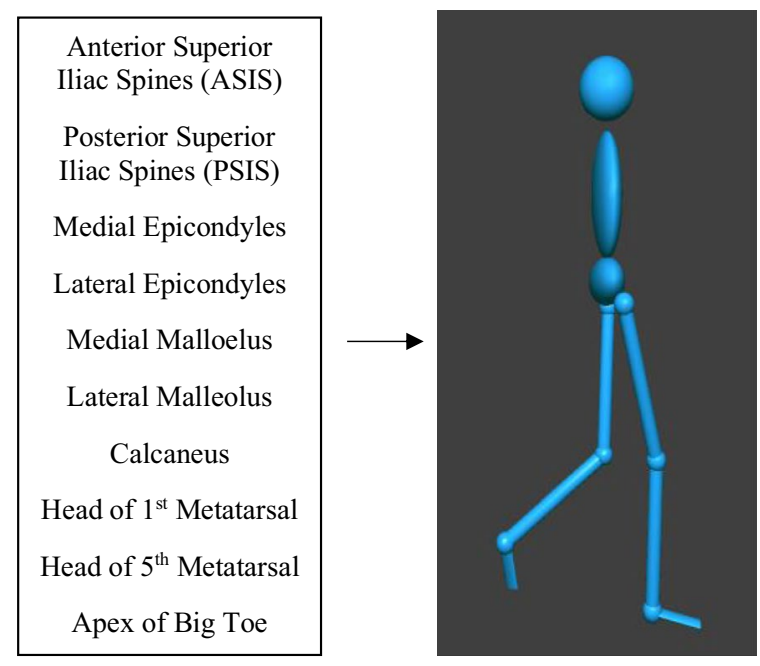

and coordination. The programme specifically developed balance using four multi-joint and complex exercises (Table 1). All exercises were performed facing forward with hands on iliac crests or relaxed by side. The training was adapted for the inclusion of the visualisation which created a non-immersive, third person perspective virtual environment. The VIS and NO-VIS exercises were very similar to allow a direct comparison as to the effect of the visualisations.

During training, all participants received verbal feedback using clear and concise cues (i.e. take pelvis straight to floor during lunge, do not cross legs during leaps, control your leg in the single leg balance). The researcher was aware of the potential for performance bias and remained as objective as possible so as not to treat the groups differently during training.

Each exercise was performed at every training session at light-moderate intensity with an expected rate of perceived exertion (RPE) of 10-12 [36, 38, 39]. Exercises were progressed as movement quality for the exercise at the given level was achieved (Table 1) [11-13, 37].

Participants wore comfortable clothing that was suitable for rehabilitative practice, and all testing procedures and training exercises were performed barefoot.

\subsection{Outcomes}

Feasibility was recorded as rate of recruitment, retention, and adherence to the training intervention. Safety was recorded as the number of adverse events during testing and/or training. Risks included ankle or other musculoskeletal injury from physical activity related to the stability training or falls during training in the laboratory. As in current clinical practice, the training was designed to be progressive enabling participants to stay within their capabilities, and participants were instructed to not perform exercises at levels beyond their ability. Falls risk was mitigated by ensuring the testing, and training space was kept clear of hazards.

Efficacy was reported through the effect of visualisation on dynamic stability, perception of stability, and rehabilitative experience.

At baseline, participants performed the Star Excursion Balance Test (SEBT) and CAIT. At the post-training testing session, the SEBT was reassessed, and the CAIT and Physical Activity Enjoyment Scale (PACES-8) questionnaire was completed.

\subsubsection{Star Excursion Balance Test}

The SEBT is an accepted and reliable method adopted in clinical practice to assess the outcome of stability-related interventions [33, 44-48].

While maintaining unilateral stance, eight maximal reaches were performed (Fig. 3) with each reach normalised to participant leg length and the maximum reach distance for all directions recorded. No weight transfer could occur during each maximal reach [47]. Failure to comply with the verbal instructions meant the trial was discarded and repeated. Up to four practice trials, each side and one test trial, were performed [49, 50].

The change in maximal reach distance from pre- to post-test for the eight reach directions, and average of the eight, was analysed. In cases of bilateral CAI, the ankle perceived as more unstable was analysed. A recent metaanalysis reported a minimal detectable change (MDC) of greater than $8.15 \%$ in the PM direction to identify success of a 4-week balance rehabilitation programme [51]. No study has identified the MDC for all eight directions of the SEBT in the CAI population. 


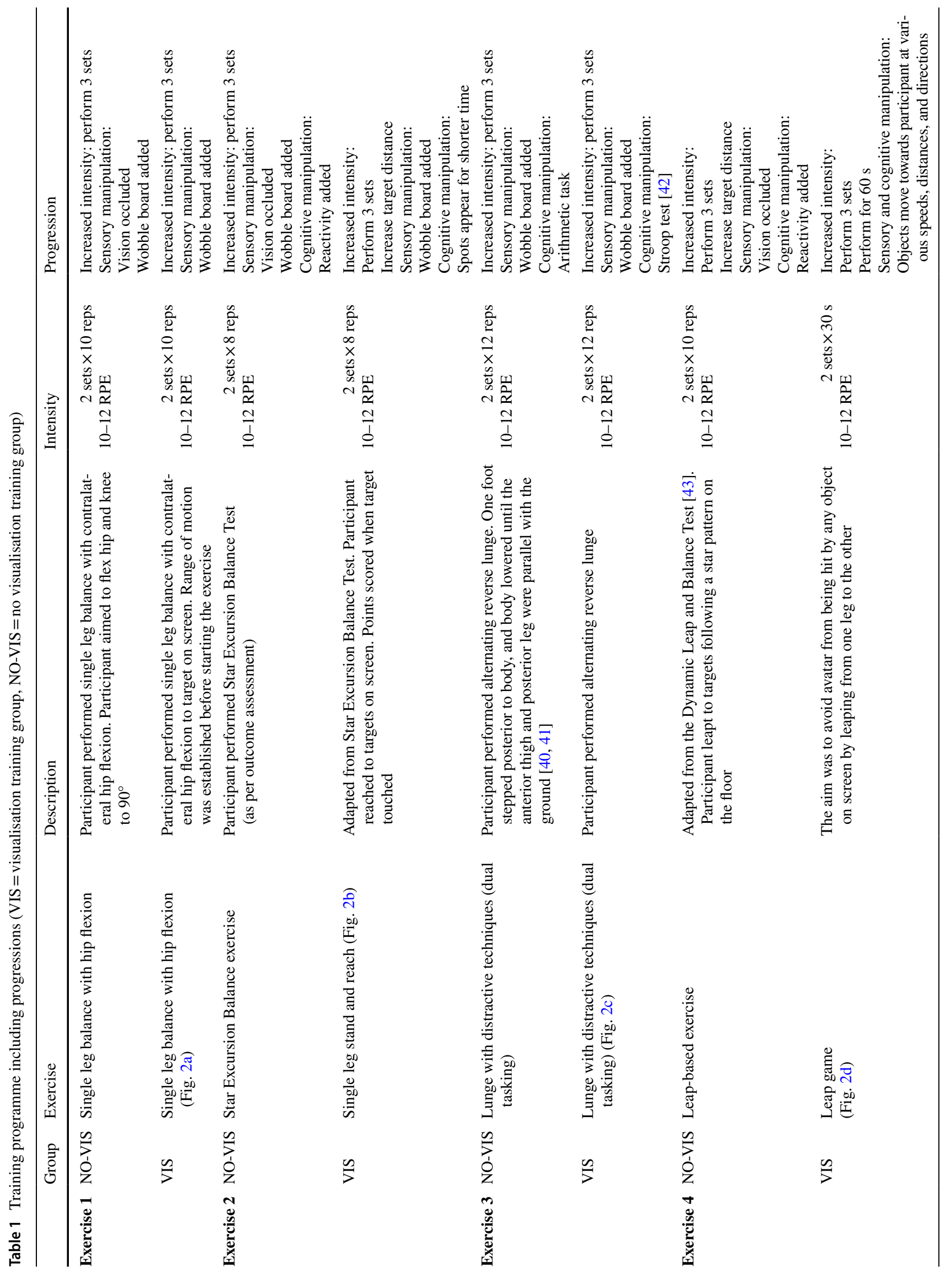




\subsubsection{Cumberland Ankle Instability Tool}

The CAIT quantified perception of stability. The minimal detectable change and minimal clinically important difference of the CAIT score is $\geq 3$ [52].

\subsubsection{Physical Activity Enjoyment Scale (PACES-8) questionnaire}

The PACES- 8 was used to quantify user experience. This measure has been frequently used in rehabilitation research into VR and balance [53-57], however not specifically in a CAI population.

The eight-item questionnaire used a five-point Likert Scale to evaluate the participants' level of enjoyment -1 being 'strongly disagree' and 5 being 'strongly agree', giving a total score out of 40. A high overall score signified high enjoyment of the training.

\subsection{Randomisation}

Participants were randomly assigned to the NO-VIS or VIS training group using a random number generator [58]. This was generated and allocated by the lead researcher.

\subsection{Blinding}

Participants and tester remained blind to group allocation until after the pre-training test. Thereafter, neither was blind to the intervention group.

\subsection{Statistical analysis}

All outcomes relating to the feasibility of the study are descriptively presented. Group data are presented as group means and standard deviation.

Statistical analysis was performed using SPSS (SPSS Statistics: v. 26, IBM, USA). Shapiro-Wilk normality tests were conducted to test for the assumption of normality.

To test if the visualisation improved performance more than stability training alone, the SEBT performance and CAIT were compared using an ANCOVA. The dependent variable was the post-test scores and the independent variable the groups (experimental and control). The pretest scores acted as a covariate to control for any differences pre-training. The PACES- 8 questionnaire was analysed using a 2-sample $t$-test. All tests were analysed at a 0.05 level of significance. The magnitude of the effects was calculated and interpreted using Cohen's effect size recommendations $(d)$, i.e. small: $0.3-0.49$; medium: $0.5-0.79$; large $\geq 0.8[59]$.
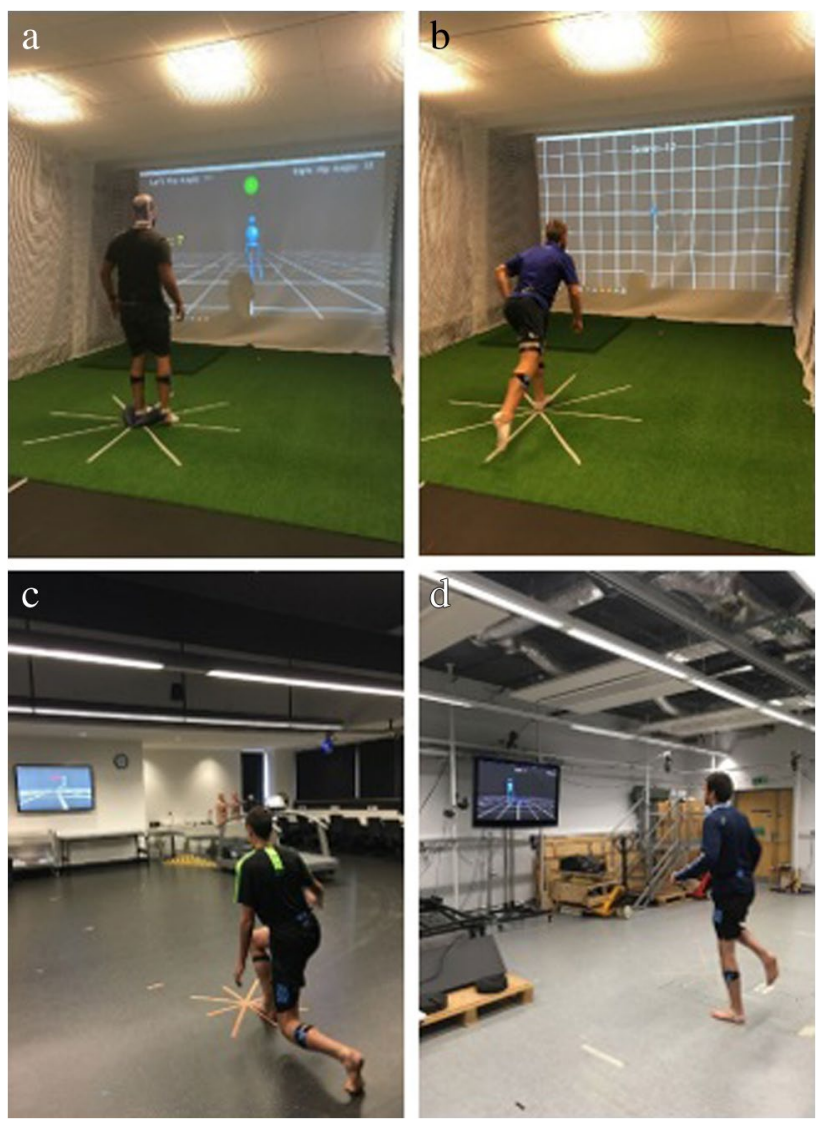

Fig. 2 a-d Examples of stability-based training exercises with visualisation

\section{Results}

From the 129 people assessed for eligibility of inclusion criteria over 4 months in the UK and 1.5 months in Australia, 17 were recruited for the study. This equated to 0.38 and 1.83 participants per week for each site, respectively. The main reason for not participating in the study were people not responding to correspondence after receiving the participant information sheet. Secondary to this was the study requiring a larger time commitment than could be given, no reimbursement for participation, and not meeting the inclusion criteria. This included revealing instability around ankle but no previous ankle injuries, lower limb dislocation, breaks, fractures, and/or surgeries, and recent significant ankle sprains. There were two dropouts during the study - an Achilles injury (VIS group) and an acute injury to the unstable ankle (NO-VIS group). Both were unrelated to the study and prevented continuation of participation. There were no adverse events related to the treatment allocations. The final analysis included 15 participants - an $88 \%$ retention rate (Fig. 4). 


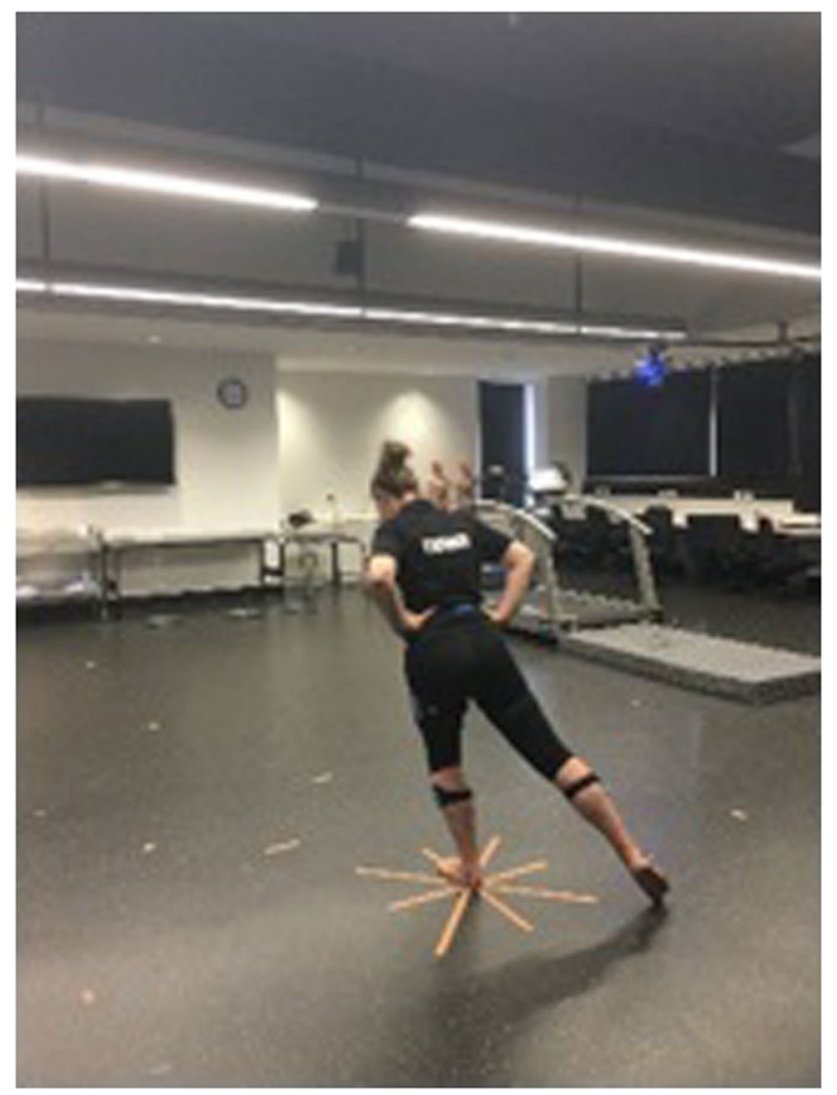

Fig. 3 Example of SEBT being performed by participant and tape to guide the reaches

Attendance at the supervised training sessions was 100\% for participants in both the VIS and No-VIS groups. There were no adverse events reported for either training group.

Descriptive statistics are presented in Table 2. There were no between-group differences in population demographics of the VIS and NO-VIS training groups ( $p \geq 0.1)$.

The results of the SEBT showed a greater increase in performance for the VIS group in the posterior-lateral (PL), and lateral (L) directions with large effect ( $d=1.5-1.8$, Table 3$)$. The greatest improvements occurred in the PL and L directions, with improvements of $12.42 \%$ and $10.04 \%$ in the VIS group, respectively, compared to improvements of $0.33 \%$ and $0.56 \%$, respectively, in the No-VIS group. The VIS group also showed a greater improvement in average reach distance $(6.74 \%)$, compared to the NO-VIS group $(0.13 \%)$, with a large effect size $(d=1.7, p=0.02$, Table 3$)$.

There were no statistically significant between group mean differences for CAIT $(d=-0.14, p=0.36)$ (Fig. 5). The CAIT score in each group individually showed clinically meaningful improvements (VIS $=3.6 \pm 3.3$ points, NOVIS $=4.2 \pm 5.3$ points).

For enjoyment, the VIS group did not score significantly higher than the NO-VIS group ( $d=0.6, p=0.26)$ (Fig. 6).

\section{Discussion}

This pilot randomised controlled trial assessed the feasibility, safety, and efficacy of stability-based training with visualisation for people with CAI. The study supports the feasibility of the 4-week stability-based training programme with visualisation. The statistically significant between group difference for objective stability, and meaningful change in subjective stability, support the potential efficacy of this training.

The protocol and training intervention using visualisation was shown to be feasible as indicated by the retention and adherence of participants. Safety of the programme was evidenced by no adverse events reported. This suggests that the design and progression of the training was an appropriate intensity, and the visualisations did not present any harm.

\subsection{Dynamic stability and perception of stability}

The stability-based training with visualisation enhanced performance of the SEBT in the lateral and posterior-lateral directions with large effect. Average reach distance was also improved. The improved reach in the posterior-lateral and lateral directions of the SEBT for the VIS group exceeded the MDC [51, 60]. This was not apparent in the NO-VIS group. Research has suggested reaching posteriorly to the body requires an increased reliance on somatosensory feedback due to lack of visual awareness. This would indicate an increased level of stability is required to maintain position [61]. Considering this, it is promising for potential future implementation of visualisation into rehabilitation practice that the greatest effect of the training occurred in these directions where remaining stable is more challenging. Moreover, to perform maximal reaching in the posterior-lateral direction, there are limited options for the body to position itself, mainly the trunk, above the base of support as opposed to when performing reaches in the medial-oriented directions. Improvements in this direction may suggest that the external focus created by the visualisation both challenged and motivated the participants to find a method to achieve the task that resulted in greater reaching distances when visualisations were removed. Meldrum and colleagues [62] reported that VR training created an external focus which provided a distraction for when practices were repetitive. Augmented feedback and/or an external focus of attention have previously been reported to elicit greater learning effects than an internal focus [14, 15, 63].

Despite improvements in the particular directions discussed above, there were limited effects in the remaining directions. Previous research has highlighted joint range of motion (ROM), and strength has been shown to significantly effect SEBT performance [64-68]. Specifically, 
Fig. 4 CONSORT flow diagram for participants

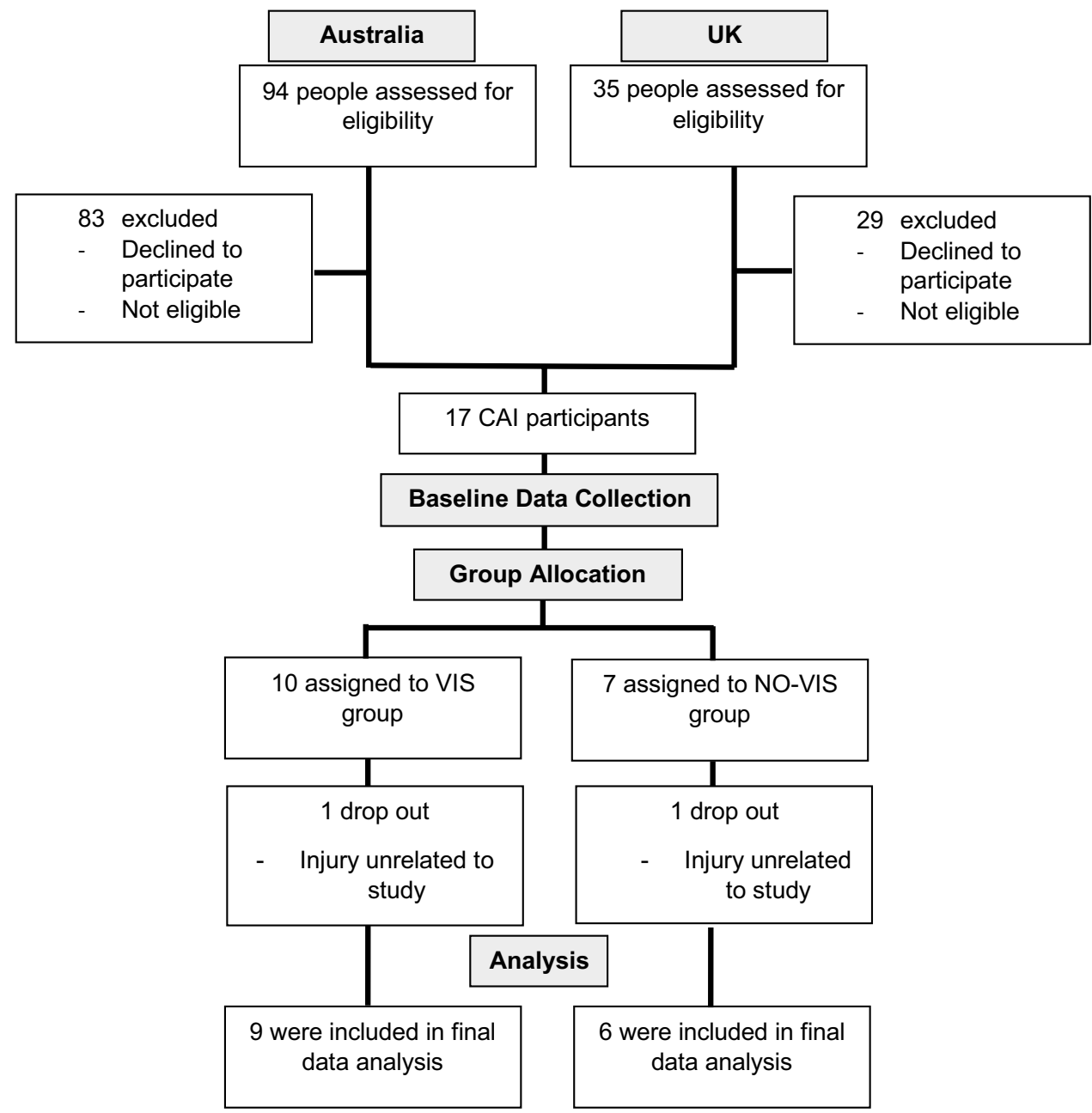

ankle dorsiflexion and knee flexion predicts anterior reach distances more than posterior reach directions [64, 65, 69]. Anterior reach distance has also been related to quadriceps muscle strength to control knee flexion during this task [69].

Table 2 Descriptive statistics (mean \pm SD) for all participants

\begin{tabular}{lll}
\hline & VIS & NO-VIS \\
\hline Site (UK/Aus) & $4 / 5$ & $2 / 4$ \\
Age (years) & $28 \pm 9$ & $29 \pm 14$ \\
Gender (M/F) & $5 / 4$ & $3 / 3$ \\
Body mass (kg) & $78.9 \pm 37.3$ & $72.1 \pm 9.6$ \\
Dominant limb (R/L) & $9 / 0$ & $6 / 0$ \\
Physically active (\%) & 77.8 & 100 \\
Competitive athlete (\%) & 22.2 & 66.7 \\
Injured ankle (R/L) & $4 / 5$ & $2 / 4$ \\
Number of ankle sprains & $3.7 \pm 2.8$ & $3.5 \pm 1.8$ \\
Treatment sought (\%) & 77.8 & 66.7 \\
Rehabilitation undertaken (\%) & 77.8 & 83.3 \\
Rehabilitation satisfaction (\%) & 44.4 & 50 \\
\hline
\end{tabular}

The current programme did not include any specific training to increase ROM or strength. This may in part explain why no improvements in anterior reach distance were observed with the training program. For both the VIS and NO-VIS groups, the improvements in perception of stability following training were of clinical significance [52]. This suggests that the stability-based training interventions both with and without visualisation for people with CAI could be used to improve perception of stability. This supports the previous research that stability-based training is an effective method of rehabilitation $[4,9,21]$.

\subsection{Rehabilitation experience}

Rehabilitation programs require adherence to be effective [21], which is more likely if the programme is enjoyable and the participants are motivated [24]. In this study, participants in both the VIS and NO-VIS groups scored enjoyment highly in the PACES-8 questionnaire. A difference in enjoyment of training between the VIS and NO-VIS groups was hypothesised. Had the NO-VIS training protocol exactly 
Table 3 Mean (SD) difference between pre- and post-test results for the SEBT

\begin{tabular}{|c|c|c|c|c|c|c|c|}
\hline \multirow[b]{2}{*}{ Reach direction } & \multicolumn{2}{|l|}{ VIS } & \multicolumn{2}{|l|}{ NO-VIS } & \multirow[b]{2}{*}{$\begin{array}{l}\text { Mean difference } \\
\text { ( } 95 \% \text { confidence intervals) }\end{array}$} & \multirow[b]{2}{*}{$d$} & \multirow[b]{2}{*}{$p$} \\
\hline & $\begin{array}{l}\text { Pre } \\
\text { (\% leg length) }\end{array}$ & Post & Pre & Post & & & \\
\hline Anterior & $66.28(5.6)$ & $67.05(5.3)$ & $68.13(5.7)$ & $66.96(7.0)$ & $1.94(-3.24$ to 5.84$)$ & 0.53 & 0.54 \\
\hline Anterior-medial & $63.77(5.1)$ & $66.08(4.4)$ & $66.43(5.5)$ & $66.43(6.1)$ & 2.31 (3.24 to 5.84$)$ & 0.53 & 0.54 \\
\hline Medial & $58.23(6.6)$ & $63.63(6.3)$ & $61.58(7.8)$ & $62.38(6.1)$ & $4.61(-3.72$ to 9.04$)$ & 0.67 & 0.38 \\
\hline Posterior-medial & $52.58(9.7)$ & $61.01(5.9)$ & $58.40(9.4)$ & $59.37(9.2)$ & $7.45(-0.68$ to 10.89$)$ & 1.29 & 0.08 \\
\hline Posterior & $46.29(11.4)$ & $57.25(5.7)$ & $53.21(10.4)$ & $54.69(7.7)$ & $9.49(-1.33$ to 11.76$)$ & 1.16 & 0.11 \\
\hline Posterior-lateral & $41.09(8.6)$ & $53.51(8.0)$ & $49.91(9.8)$ & $50.24(7.3)$ & $12.10(0.72$ to 15.77$)$ & 1.82 & $0.03 *$ \\
\hline Lateral & $39.52(8.7)$ & $49.56(7.6)$ & $46.28(9.4)$ & $46.85(7.5)$ & $9.48(0.55$ to 13.11$)$ & 1.56 & $0.04 *$ \\
\hline Anterior-lateral & $58.97(11.0)$ & $59.31(8.6)$ & $61.84(9.6)$ & $59.23(6.4)$ & $2.95(-5.30$ to 8.49$)$ & 0.39 & 0.624 \\
\hline Average & $52.97(6.3)$ & $59.72(5.3)$ & $58.14(7.1)$ & $58.27(5.2)$ & $6.61(0.86$ to 8.78$)$ & 1.69 & $0.02^{*}$ \\
\hline
\end{tabular}

*Significant difference between VIS and NO-VIS groups $(p \leq 0.05)$

Fig. 5 Pre- and post-test CAIT score for the VIS and NO-VIS groups

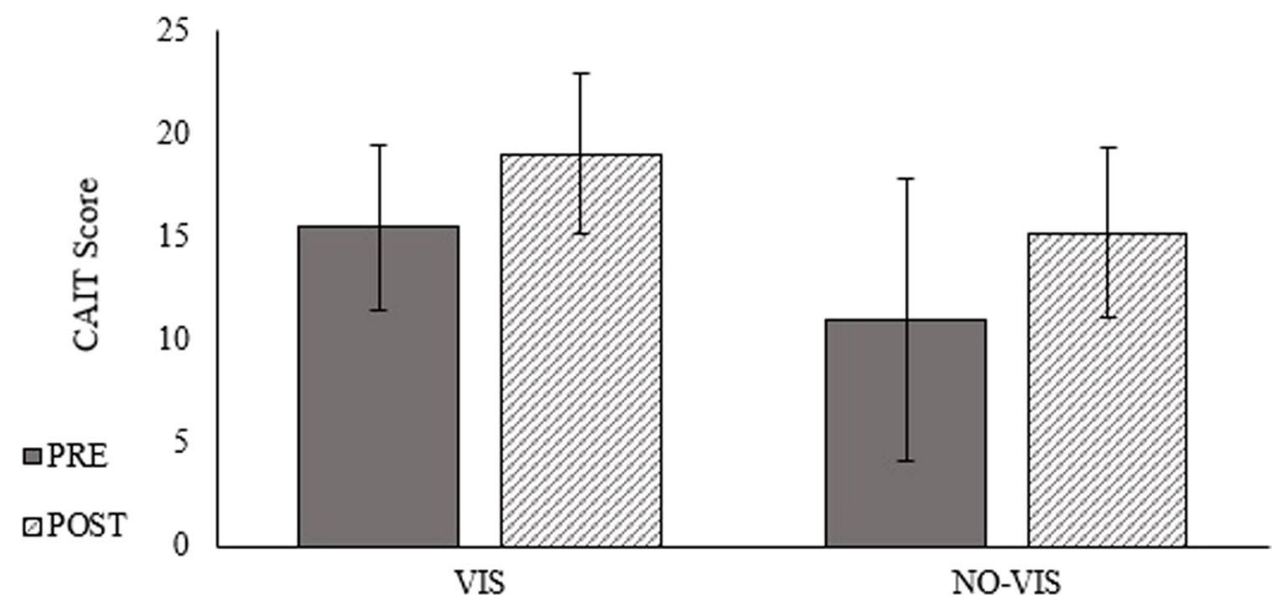

followed that of routine clinical practice, current ankle treatment guidelines, or a programme from a previous study, as opposed to the training programme conducted here, it is believed the difference of enjoyment reported between

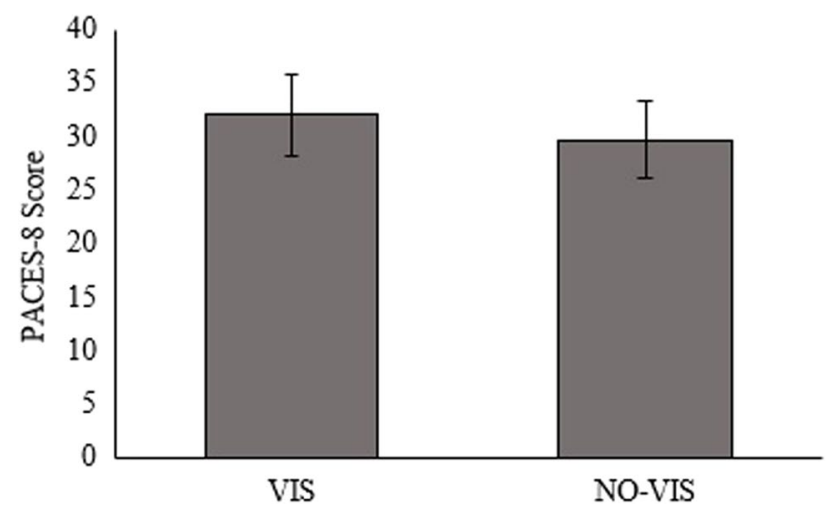

Fig. 6 Perception of enjoyment of training for the VIS and NO-VIS groups groups would have been greater. Like the VIS group, the NO-VIS training was designed as a more functional and progressive programme to those seen before, just without the visualisation. Furthermore, the laboratory environment and motion capture equipment may have also influenced the rehabilitative experience. Both groups trained in the laboratory environment, thus, it is possible a difference in enjoyment may have been evident had the control group participated in an unsupervised or at home rehabilitation programme where the environment was not changed and supervision not present. For future work, it would be interesting to examine the enjoyment of a programme completed at home, and not in a clinical or laboratory-based setting.

For both groups, a large majority of participants $(\geq 50 \%)$ were previously unsatisfied with their rehabilitation. In the current study, participants in both groups reported clinically meaningful improvements in perceived stability, and this may have increased confidence and trust in the program. This would satisfy both the need for competence and relatedness. In turn, this may have increased motivation in both 
groups, leading to increased effort and engagement [23, 24]. Although this may have been subconscious, this could have resulted in further perceived improvements leading to greater enjoyment from the programme, as it was perceived more successful than previous experiences, and ultimately resulting in only a small difference between VIS and NOVIS enjoyment scores.

\section{Study limitations}

There are limitations to this study. Firstly, despite the sample population representing a diverse group, the sample size was small, and the randomization of participants led to unequal numbers in each of the groups. Therefore, any inferences from the results should be interpreted with caution. Participant recruitment was impacted by the multi-centre nature of the study. Given the study was conducted internationally across three sites, a new participant recruitment protocol was required each time. However, this is believed to strengthen the study, despite the small sample size, since the protocol was completed in three different locations with no defining impact on retention, adherence, and safety. Given the limited research regarding chronic ankle instability and use of visualisation, the study was designed to assess initial feasibility to establish whether further study would be warranted with a larger sample size, and it is believed this has been achieved. It is important for future work to consider the recruitment rate. Primarily, this should include increased follow up of people who have expressed interest in the study. Further, researchers could consider providing reimbursement to participants. Reducing the time commitment may also be considered, but this may influence efficacy, and the protocol used in this study aimed to represent current clinical practice and research [33-36, 39].

After allocating the participants to the VIS or NO-VIS group following baseline testing, the participants were no longer blinded as to the training group they were part of. Due to the nature of the study this was not possible but is a limitation presenting possible bias. For the testing and training, the lead researcher was the only tester, subjecting the study to further potential bias. However, having only one tester allowed for consistent practice throughout testing and training as would occur in rehabilitative practice.

Due to the nature of the study, supervised laboratory visits were required for study completion. Participants attended all training sessions, but future studies could aim to monitor adherence to visualisation during unsupervised or homebased training scenarios, if facilities and equipment permit. Further to this, future research should use a specific measure for motivation to analyse the specific type of motivation the training may have created relative to the self-determination theory [70] continuum.
This study was sufficiently powered for the SEBT. Based on the results of this study, an estimated sample size of 16 per group would be required for future work to assess enjoyment with visualisations included in the training programme and for the CAIT 62 per group. This was based on the results of the current study at a power of 0.80 and alpha level of 0.05 . To be adequately powered for all outcomes, the minimum sample size would be 62, not accounting for dropouts. Future studies could also examine additional or alternative outcome methods of perceived stability, such as the Foot and Ankle Ability Measure [71], as well as conducting short interviews for more in-depth analysis.

\section{Conclusion}

The results of this pilot study support the feasibility and safety of stability-based training with visualisation in those with CAI. We found enhanced performed on the SEBT when training with visualisation which suggests this could be an effective approach to stability-based training. Further investigation using a larger sample and additional subjective measures is needed to thoroughly assess stability and enjoyment when visualisation is incorporate into training.

Open Access This article is licensed under a Creative Commons Attribution 4.0 International License, which permits use, sharing, adaptation, distribution and reproduction in any medium or format, as long as you give appropriate credit to the original author(s) and the source, provide a link to the Creative Commons licence, and indicate if changes were made. The images or other third party material in this article are included in the article's Creative Commons licence, unless indicated otherwise in a credit line to the material. If material is not included in the article's Creative Commons licence and your intended use is not permitted by statutory regulation or exceeds the permitted use, you will need to obtain permission directly from the copyright holder. To view a copy of this licence, visit http://creativecommons.org/licenses/by/4.0/.

\section{References}

1. Gribble PA, Bleakley CM, Caulfield BM, Docherty CL, Fourchet F, Fong DTP, Hertel J, Hiller CE, Kamaninski TW, McKeon PO, Refshauge KM, Verhagen EA, Vicenzino BT, Wikstrom EA, Delahunt E (2016) Evidence review for the 2016 International Ankle Consortium consensus statement on the prevalence, impact and long-term consequences of lateral ankle sprains. Br J Sports Med 50:1496-1505

2. Hiller CE, Nightingale EJ, Raymond J, Kilbreath SL, Burns J, Black DA, Refshauge KM (2012) Prevalence and impact of chronic musculoskeletal ankle disorders in the community. Arch Phys Med Rehabil 93:1801-1807

3. Blalock D, Miller A, Tilley M, Wang J (2015) Joint instability and osteoarthritis. Clin Med Insights Arthritis Musculoskelet Disord 8:15-23

4. Kosik KB, Johnson NF, Terada M, Thomas AC, Mattacola CG, Gribble PA (2019) Decreased dynamic balance and dorsiflexion 
range of motion in young and middle-aged adults with chronic ankle instability. J Sci Med Sport 22:976-980

5. Golditz T, Steib S, Pfeifer K, Uder M, Gelse K, Janka R, Hennig FF, Welsch GH (2014) Functional ankle instability as a risk factor for osteoarthritis: using T2-mapping to analyze early cartilage degeneration in the ankle joint of young athletes. Osteoarthritis Cartilage 22:1377-1385

6. Saltzman C, Salamon M, Blanchard M, Huff T, Hayes A, Buckwalter J, Amendola A (2005) Epidemiology of ankle arthritis: report of a consecutive series of 369 patients from a tertiary orthopaedic centre. Iowa Orthop J 25:44-46

7. Gramlich Y, Neun O, Klug A, Buckup J, Stein T, Neumann A, Fischer S, Abt HP, Hoffmann R (2018) Total ankle replacement leads to high revision rates in post-traumatic end-stage arthrosis. Int Orthop 42:2375-2381

8. Ramaskandhan J, Rashid A, Kometa S, Siddique MS (2020) Comparison of 5-year patient-reported outcomes (PROMs) of total ankle replacement (TAR) to total knee replacement (TKR) and total hip replacement (THR). Foot Ankle Int 41:767-774

9. Guillodo Y, Goff A, Saraux A (2011) Adherence and effectiveness of rehabilitation in acute ankle sprain. Ann Phys Rehabil Med 54:225-235

10. Li Z, Han XG, Sheng J, Ma SJ (2016) Virtual reality for improving balance in patients after stroke: a systematic review and meta-analysis. Clin Rehabil 30:432-440

11. Chodzko-Zajko WJ, Proctor DN, Singh MAF, Minson CT, Nigg CR, Salem GJ, Skinner JS (2009) Exercise and physical activity for older adults. Med Sci Sports Exerc 41:1510-1530

12. Dault MC, Geurts ACH, Mulder TW, Duysens J (2001) Postural control and cognitive task performance in healthy participants while balancing on different support-surface configurations. Gait Posture 14:248-255

13. Klatt B, Carender W, Lin C, Alsubaie S, Kinnaird C, Sienko K, Whitney S (2015) A conceptual framework for the progression of balance exercises in persons with balance and vestibular disorders. Int J Phys Med Rehabil 2

14. Rotem-Lehrer N, Laufer Y (2007) Effect of focus of attention on transfer of a postural control task following an ankle sprain. J Orthop Sports Phys Ther 37:564-569

15. Welling W, Benjaminse A, Gokeler A, Otten B (2016) Enhanced retention of drop vertical jump landing technique: a randomized controlled trial. Hum Mov Sci 45:84-95

16. Vogt S, Kjaeret-Maroni N, Neuhaus D, Baumeister J (2019) Virtual reality interventions for balance prevention and rehabilitation after musculoskeletal lower limb impairments in young up to middle-aged adults: a comprehensive review on used technology, balance outcome measures and observed effects. Int $\mathbf{J}$ Med Informatics 126:46-58

17. Howard MC (2017) A meta-analysis and systematic literature review of virtual reality rehabilitation programs. Comput Hum Behav 70:317-327

18. Kalron A, Fonkatz I, Frid L, Baranshi H, Achiron A (2016) The effect of balance training on postural control in people with multiple sclerosis using the CAREN virtual reality system: a pilot randomized controlled trial. J Neuroeng Rehabil 13

19. Dos Santos LF, Christ O, Mate K, Schmidt H, Kruger J, Dohle C (2016) Movement visualisation in virtual reality rehabilitation of the lower limb: a systematic review. Biomed Eng Online 15

20. Rose T, Nam CS, Chen KB (2018) Immersion of virtual reality for rehabilitation - review. Appl Ergon 69:153-161

21. Argent R, Daly A, Caulfield B (2018) Patient involvement with home-based exercise programs: can connected health interventions influence adherence? Jmir Mhealth Uhealth 6

22. Campbell R, Evans M, Tucker M, Quilty B, Dieppe P, Donovan JL (2001) Why don't patients do their exercises? Understanding non-compliance with physiotherapy in patients with osteoarthritis of the knee. J Epidemiol Commun Health 55:132-138

23. Carson F, Polman RCJ (2017) Self-determined motivation in rehabilitating professional rugby union players. Bmc Sports Sci Med Rehabil 9

24. Marshall A, Donovan-Hall M, Ryall S (2012) An exploration of athletes' views on their adherence to physiotherapy rehabilitation after sport injury. J Sport Rehabil 21:18-25

25. Teixeira PJ, Carraca EV, Markland D, Silva MN, Ryan RM (2012) Exercise, physical activity, and self-determination theory: a systematic review. Int J Behav Nutr Phys Act 9

26. Ballinger C, Taylor A, Loudon D, Macdonald AS (2016) ) Rehabilitation professionals' perceptions of the use of new visualisation software tools with people with stroke. Disabil Rehabil Assist Technol 11:139-149

27. Gribble PA, Delahunt E, Bleakley CM, Caulfield B, Docherty CL, Fong DTP, Fourchet F, Hertel J, Hiller CE, Kaminski TW, McKeon PO, Refshauge KM, Van Der Wees P, Vicenzino W, Wikstrom EA (2014) Selection criteria for patients with chronic ankle instability in controlled research: a position statement of the international ankle consortium. J Athl Train 49:121-127

28. Hiller CE, Refshauge KM, Bundy AC, Herbert RD, Kilbreath SL (2006) The cumberland ankle instability tool: a report of validity and reliability testing. Arch Phys Med Rehabil 87:1235-1241

29. Millar LJ, Meng L, Rowe PJ (2019) Routine clinical motion analysis: comparison of a bespoke real-time protocol to current clinical methods. Comput Methods Biomech Biomed Eng 22:149-158

30. Tawy GF, Rowe P (2017) Is the instrumented-pointer method of calibrating anatomical landmarks in 3D motion analysis reliable? J Biomech 53:205-209

31. Grood ES, Suntay WJ (1983) A joint coordinate system for the clinical description of 3-dimensional motions - application to the knee. J Biomech Eng Trans ASME 105:136-144

32. Wu G, Cavanagh PR (1995) ISB recommendations for standardization in the reporting of kinematic data. J Biomech 28:1257-1260

33. Anguish B, Sandrey MA (2018) Two 4-week balance-training programs for chronic ankle instability. J Athl Train 53:662-671

34. Clark NC, Roijezon U, Treleaven J (2015) Proprioception in musculoskeletal rehabilitation. Part 2: Clinical assessment and intervention. Man Ther 20:378-387

35. Cruz-Diaz D, Lomas-Vega R, Osuna-Perez MC, Contreras FH, Martinez-Amat A (2015) Effects of 6 weeks of balance training on chronic ankle instability in athletes: a randomized controlled trial. Int J Sports Med 36:754-760

36. Hupperets MDW, Verhagen E, Van Mechelen W (2009) Effect of unsupervised home based proprioceptive training on recurrences of ankle sprain: randomised controlled trial. Br Med J 339

37. American College of Sports Medicine (ACSM) (2018) ACSM's guidelines for exercise testing and prescription. Wolters Kluwer, Philadelphia

38. Borg G (1998) Borg's perceived exertion and pain scales. Human Kinetics, Champaign

39. Hale SA, Fergus A, Axmacher R, Kiser K (2014) Bilateral improvements in lower extremity function after unilateral balance training in individuals with chronic ankle instability. J Athl Train 49:181-191

40. Nadzalan AM, Mohamad NI, Low JFL, Ahmad R, Waqqash E (2017) Fascicle behaviour analysis during forward lunge exercise: the comparisons between training loads. J Fundam Appl Sci 9:1090-1101

41. Kritz M, Cronin J, Hume P (2009) Using the body weight forward lunge to screen an athlete's lunge pattern. Strength Cond J $31: 15-24$ 
42. Stroop JR (1935) Studies of interference in serial verbal reactions. J Exp Psychol 18:643-662

43. Jaffri AH, Newman TM, Smith BI, Miller SJ (2017) The dynamic leap and balance test (DBLT): a test-retest reliability study. Int J Sports Phys Ther 12:512-519

44. Delahunt E, Bleakley CM, Bossard DS, Caulfield BM, Docherty CL, Doherty C, Fourchet F, Fong DT, Hertel J, Hiller CE, Kaminski TW, McKeon PO, Refshauge KM, Remus A, Verhagen E, Vicenzino BT, Wikstrom EA, Gribble PA (2018) Clinical assessment of acute lateral ankle sprain injuries (ROAST): 2019 consensus statement and recommendations of the International Ankle Consortium. Br J Sports Med 52:1304-1310

45. McKeon PO, Hertel J (2008) Systematic review of postural control and lateral ankle instability, part II: Is balance training clinically effective? J Athl Train 43:305-315

46. Wan Y, Davies JL, Button K, Al-Amri M (2019) Effect of visual feedback on the performance of the Star Excursion Balance Test. J Rehabil Assist Technol Eng 6

47. Gribble PA, McLeod MM, Pietrosimone BG (2012) Influence of lower extremity kinematics on dynamic stability in patients with chronic ankle instability. Med Sci Sports Exerc 44:201-201

48. Ness BM, Comstock BA, Schweinle WE (2016) Changes in dynamic balance and hip strength after an eight-week conditioning program in NCAA Division I female soccer (football) athletes. Int J Sports Phys Ther 11:1054-1064

49. Pionnier R, Decoufour N, Barbier F, Popineau C, SimimoneauBuessinger E (2016) A new approach of the Star Excursion Balance Test to assess dynamic postural control in people complaining from chronic ankle instability. Gait Posture 45:97-102

50. Robinson R, Gribble P (2008) Support for a reduction in the number of trials needed for the Star Excursion Balance Test. Arch Phys Med Rehabil 89:364-370

51. Burcal CJ, Sandrey MA, Hubbard-Turner T, McKeon PO, Wikstrom EA (2019) Predicting dynamic balance improvements following 4-weeks of balance training in chronic ankle instability patients. J Sci Med Sport 22:538-543

52. Wright CJ, Linens SW, Cain MS (2017) Establishing the minimal clinical important difference and minimal detectable change for the Cumberland Ankle Instability Tool. Arch Phys Med Rehabil 98:1806-1811

53. Padala K, Padala P, Lensing S, Dennis R, Bopp M, Parkes C, Garrison M, Dubbert P, Roberson P, Sullivan D (2017) Efficacy of Wii-Fit on static and dynamic balance in community dwelling older veterans: a randomized controlled pilot trial. J Aging Res 9

54. Teques P, Calmeiro L, Silva C, Borrego C (2017) Validation and adaptation of the Physical Activity Enjoyment Scale (PACES) in fitness group exercisers. J Sport Health Sci

55. Tripette J, Murakami H, Ryan KR, Ohta Y, Miyachi M (2017) The contribution of Nintendo Wii Fit series in the field of health: a systematic review and meta-analysis. Peerj 5

56. Van Diest M, Stegenga J, Wortche HJ, Postema K, Verkerke GJ, Lamoth CJC (2014) Suitability of Kinect for measuring whole body movement patterns during exergaming. J Biomech 47:2925-2932

57. Vaziri DD, Aal K, Ogonowski C, Von Rekowski T, Kroll M, Martson HR, Poveda R, Gschwind YJ, Delbaere K, Wieching R, Wulf V (2016) Exploring user experience and technology acceptance for a fall prevention system: results from a randomized clinical trial and a living lab. Eur Rev Aging Phys Act 13

58. RANDOM.ORG (2020) Random Sequence Generator [Online]. Available: https://www.random.org/sequences/ [Accessed 01/04/2018]

59. Cohen J (1988) Statistical power analysis for the behavioural sciences. Lawrence Eribaum, New Jersey
60. Hoch M, Gaven S, Weinhandl J (2016) Kinematic predictors of Star Excursion Balance Test performance in individuals with chronic ankle instability. Clin Biomech 35

61. Bulow A, Anderson JE, Leiter JR, Macdonald PB, Peeler J (2019) The modified Star Excursion Balance and Y-Balance Test results differ when assessing physically active healthy adolescent females. Int J Sports Phys Ther 14:192-203

62. Meldrum D, Glennon A, Herdman S, Murray D, McConn-Walsh R (2011) Virtual reality rehabilitation of balance: assessment of the usability of the Nintendo Wii Fit Plus. Disabil Rehabil Assist Technol 1-6

63. Keller M, Lauber B, Gottschalk M, Taube W (2015) Enhanced jump performance when providing augmented feedback compared to an external or internal focus of attention. J Sports Sci 33:1067-1075

64. Basnett CR, Hanish MJ, Wheeler TJ, Miriovsky DJ, Danielson EL, Barr JB, Grindstaff TL (2013) Ankle dorsiflexion range of motion influences dynamic balance in individuals with chronic ankle instability. Int J Sports Phys Ther 8:121-128

65. Gabriner ML, Houston MN, Kirby JL, Hoch MC (2015) Contributing factors to Star Excursion Balance Test performance in individuals with chronic ankle instability. Gait Posture 41:912-916

66. Gribble PA, Hertel J, Denegar CR (2007) Chronic ankle instability and fatigue create proximal joint alterations during performance of the Star Excursion Balance Test. Int J Sports Med 28:236-242

67. Gribble PA, Hertel J, Denegar CR, Buckley WE (2004) The effects of fatigue and chronic ankle instability on dynamic postural control. J Athl Train 39:321-329

68. McCann RS, Crossett ID, Terada M, Kosik KB, Bolding BA, Gribble PA (2017) Hip strength and Star Excursion Balance Test deficits of patients with chronic ankle instability. J Sci Med Sport 20:992-996

69. Pinheiro LSP, Ocarino JD, Bittencourt NFN, Souza TR, Martins SCD, Bomtempo RAB, Resende RA (2020) Lower limb kinematics and hip extensors strengths are associated with performance of runners at high risk of injury during the modified Star Excursion Balance Test. Braz J Phys Ther 24:488-495

70. Ryan RM, Deci EL (2000) Self-determination theory and the facilitation of intrinsic motivation, social development, and wellbeing. Am Psychol 55:68-78

71. Carcia CR, Martin RL, Drouin JM (2008) Validity of the foot and ankle ability measure in athletes with chronic ankle instability. J Athl Train 43:179-183

Publisher's note Springer Nature remains neutral with regard to jurisdictional claims in published maps and institutional affiliations.

Lauren Forsyth received her $\mathrm{PhD}$ in Biomedical Engineering from the University of Strathclyde in 2021. Her research interests include biomechanics, chronic ankle instability, rehabilitation, and virtual reality.

Jason Bonacci is a physiotherapist who received his $\mathrm{PhD}$ in biomechanics at The University of Queensland. He is a senior lecturer in biomechanics at Deakin University, Australia. His research focuses on biomechanics of human movement.

Dr Craig Childs is a teaching fellow at the University of Strathclyde. As manager of the CAREN lab, he supervises various projects, including perturbation-based gait assessment in amputees and older adults. 